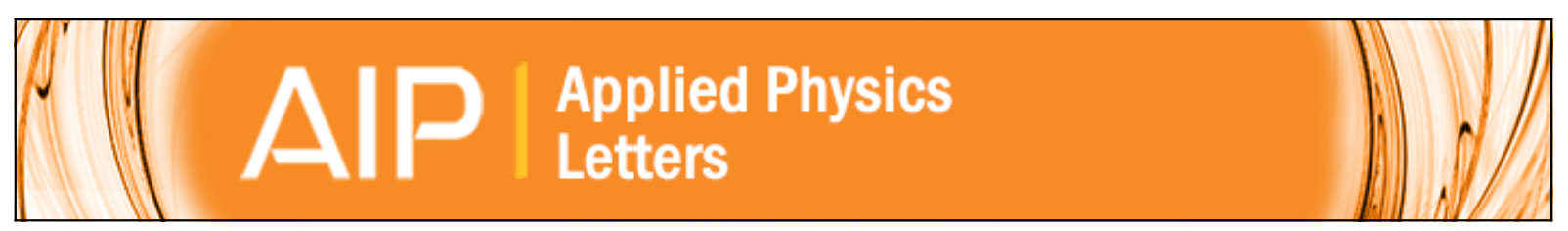

\title{
Large enhanced dielectric permittivity in polyaniline passivated core-shell nano magnetic iron oxide by plasma polymerization
}

Lija K. Joy, V. Sooraj, U. S. Sajeev, Swapna S. Nair, T. N. Narayanan, N. Sethulakshmi, P. M. Ajayan, and M. R. Anantharaman

Citation: Applied Physics Letters 104, 121603 (2014); doi: 10.1063/1.4870098

View online: http://dx.doi.org/10.1063/1.4870098

View Table of Contents: http://scitation.aip.org/content/aip/journal/apl/104/12?ver=pdfcov

Published by the AIP Publishing

\section{Articles you may be interested in}

Contact potential induced enhancement of magnetization in polyaniline coated nanomagnetic iron oxides by plasma polymerization

Appl. Phys. Lett. 103, 162414 (2013); 10.1063/1.4826459

Ion irradiation of Fe-Fe oxide core-shell nanocluster films: Effect of interface on stability of magnetic properties J. Appl. Phys. 114, 083903 (2013); 10.1063/1.4818309

Synthesis and magnetic properties of core/shell $\mathrm{FeO} / \mathrm{Fe} 3 \mathrm{O} 4$ nano-octopods

J. Appl. Phys. 113, 17B508 (2013); 10.1063/1.4794978

Facile synthesis of single-phase spherical -Fe16N2/Al2O3 core-shell nanoparticles via a gas-phase method J. Appl. Phys. 113, 164301 (2013); 10.1063/1.4798959

Synthesis of ferromagnetic nanoparticles with a core/shell structure by a self-propagating combustion method J. Appl. Phys. 109, $07 B 533$ (2011); 10.1063/1.3563062

\section{AlP Re-register for Table of Content Alerts}




\title{
Large enhanced dielectric permittivity in polyaniline passivated core-shell nano magnetic iron oxide by plasma polymerization
}

\author{
Lija K. Joy, ${ }^{1}$ V. Sooraj, ${ }^{1}$ U. S. Sajeev,${ }^{2}$ Swapna S. Nair ${ }^{3}$ T. N. Narayanan, ${ }^{4}$ \\ N. Sethulakshmi, ${ }^{1}$ P. M. Ajayan, ${ }^{5}$ and M. R. Anantharaman ${ }^{1, a)}$ \\ ${ }^{1}$ Department of Physics, Cochin University of Science and Technology, Cochin-682022, Kerala, India \\ ${ }^{2}$ Department of Physics, Government College, Kottayam-686613, Kerala, India \\ ${ }^{3}$ Department of Physics, School of Mathematical and Physical Sciences, Central University of Kerala, \\ Kasargode-671123, Kerala, India \\ ${ }^{4}$ CSIR-Central Electrochemical Research Institute, Karaikkudi-630006, Tamil Nadu, India \\ ${ }^{5}$ Department of Material Science and Nano Engineering, Rice University, 6100 Main Street, Houston, \\ Texas 7700, USA
}

(Received 21 December 2013; accepted 17 March 2014; published online 28 March 2014)

\begin{abstract}
Commercial samples of Magnetite with size ranging from $25-30 \mathrm{~nm}$ were coated with polyaniline by using radio frequency plasma polymerization to achieve a core shell structure of magnetic nanoparticle (core)-Polyaniline (shell). High resolution transmission electron microscopy images confirm the core shell architecture of polyaniline coated iron oxide. The dielectric properties of the material were studied before and after plasma treatment. The polymer coated magnetite particles exhibited a large dielectric permittivity with respect to uncoated samples. The dielectric behavior was modeled using a Maxwell-Wagner capacitor model. A plausible mechanism for the enhancement of dielectric permittivity is proposed. @ 2014 AIP Publishing LLC. [http://dx.doi.org/10.1063/1.4870098]
\end{abstract}

Nano magnetic iron oxides are of great technological importance, and they find extensive applications in the field of medicine and information storage. They are biocompatible and increasingly being employed for diagnostics, ${ }^{1}$ therapy, ${ }^{4}$ magnetic drug delivery and gene delivery in the area of medicine. ${ }^{1-4}$ Magnetite and maghemite are well-known members belonging to the family of iron oxides and they crystallize in inverse spinel structure; ${ }^{6}$ magnetite can be written in the form $\mathrm{Fe}^{3+}{ }_{A}\left[\mathrm{Fe}^{3+} \mathrm{Fe}^{2+}\right]_{B} \mathrm{O}_{4}^{2-}$ while maghemite is in the form $\mathrm{Fe}^{3+}{ }_{A}\left[\mathrm{Fe}_{\frac{5}{3}}^{3+} \Delta_{\frac{1}{3}}\right]_{B} \mathrm{O}_{4}^{2-}$, where vacancies are exclusively concentrated on the octahedral B sites. They are candidate materials for many industrial applications such as dense magnetic recording media or for the synthesis of ferro fluids and of late sought after for many biomedical applications. ${ }^{4}$ They are also important from a fundamental perspective because they are ideal ferrimagnets exhibiting size effects and transform to super paramagnetic materials and hence suitable for biomedical applications. ${ }^{5}$

For biomedical applications, a core shell architecture is preferred with the core being an iron oxide and the shell being a coating of silica or gold enabling the possibility of conjugating drugs for delivery or attaching molecule of specificity for detection. Thus, the entire regime of converting these materials for applications revolves around core-shell architectures. Normally, core-shell architectures are synthesized in situ, and these can also be prepared by an appropriate coating of an organic medium or a polymer on the iron oxide. Such coatings of polymers are considered biocompatible and increases thermal stability.

Though there are reports ${ }^{7,8}$ on the influence of polymer coating on the magnetic properties of core-shell structures,

\footnotetext{
${ }^{\text {a) }}$ Author to whom correspondence should be addressed. Electronic mail: mraiyer@yahoo.com
}

reports on the influence of shell on the overall dielectric properties of the core shell are scarce or seldom investigated. In such core shell structures consisting of a magnetic core and a polymer shell, dielectric properties can be greatly modified. It is presumed that the morphological characteristics of the core can be combined with the electrical properties of the shell, a polymer. Under such a scenario, interfacial effects play a dominant role, and the possibility of enhancement of dielectric properties of the core-shell structure appears very bright.

The advantage of having enhanced dielectric permittivity of a magnetic core-polymer shell is multifold. They can be very useful for applications in the field of solid state devices such as active electrode materials in energy storage by storing large amount of charges in the capacitors which can deliver energy instantaneously for electronic equipment. ${ }^{9-12}$ These kinds of pulse power applications are of interest to military, such as in kinetic energy weapons, electromagnetic armor, and hybrid electric vehicles. ${ }^{13}$

There exist reports in literature ${ }^{14-17}$ wherein core shell structure of iron oxide-poly pyrrole has been investigated in detail. For instance, Yang Xiaotun et al. reported that the conjugation length of poly pyrrole is not affected after being deposited on the surface of iron Oxide nano particles. ${ }^{17}$ They concluded that the electrical conductivity remained unaltered with $\gamma-\mathrm{Fe}_{2} \mathrm{O}_{3}$ content. However, it must be mentioned here that theirs was a study to look into the effect of concentration of $\gamma-\mathrm{Fe}_{2} \mathrm{O}_{3}$ on the electrical properties and the method adopted for the synthesis of composites was in situ. Tabata et al. attributed the effect of dielectric enhancement to the interlayer strain caused by the interfacial lattice mismatch in the dielectric super lattices of $\mathrm{SrTiO}_{3} / \mathrm{BaTiO}_{3} \cdot{ }^{18}$ Liu et al. reported that electric field in the core region with a shell of spherically anisotropic material is found to be enhanced when compared to an isotropic shell material. ${ }^{19}$ 
The influence of conducting polymer coating on the electrical behaviour of core nano particles can be directly modeled by Maxwell-Wagner capacitor model. ${ }^{20-23}$ Maxwell-Wagner relaxation based on extrinsic effects such as Internal Barrier Layer Capacitance ${ }^{24,25}$ (IBLC) and Surface Barrier Layer Capacitance ${ }^{26}$ (SBLC) is usually employed to explain the unusual enhanced dielectric behavior. In the IBLC scenario, insulating grain boundary layers between semiconducting grains offers a depletion layer at the interface which generates dielectric permittivity through Maxwell-Wagner relaxation. ${ }^{27}$ SBLC considers the formation of Schottky barriers at the interfaces of metal-to insulator contacts/inter grain boundaries leading to the suppression of electron concentration in the contact region of semiconductor. ${ }^{28}$ Also, by assuming the core shell structure similar to a composite arrangement consisting of two parallel-sided slabs of materials with different properties, the influence of coating thickness on dielectric permittivity can be modeled by effective medium theory. ${ }^{29-31}$ Thus, it is possible to tailor the dielectric permittivity of core-shell structure by suitable shell thickness.

The present work focuses on Polyaniline (PANI) coated on $\mathrm{Fe}_{3} \mathrm{O}_{4}$. Among other intrinsic conducting polymers, polyaniline is preferred due its oxidative stability, simple synthesis, fairly large conductivity, and easily tunable properties. ${ }^{32}$ Usually, dry and wet approaches are adopted for the conventional organic coating of nano particles. Plasma polymerization technique which comes under dry method is an inexpensive and promising method for providing pinhole-free, chemically and thermally stable uniform organic coating. ${ }^{33}$ When a material is exposed to plasma, its surface gets modified due to the chemical and physical changes on the surface during plasma treatment process. Based on the source generator for electric discharge process for plasma polymerization, it can be categorized into three forms, namely, direct current (dc), alternating current (ac), and radio frequency (rf) plasma. We employed a home made RF plasma polymerization unit for the surface modification of $\mathrm{Fe}_{3} \mathrm{O}_{4}$ nano particles. The coating thickness could be controlled by parameters such as monomer flow rate, RF power, current density, deposition time, and pressure.

Reports on $\mathrm{Fe}_{3} \mathrm{O}_{4}$-PANI composites are also in plenty. ${ }^{34-36}$ Most of the techniques adopted for the synthesis of these composite are in situ chemical methods. Mahy et al. observed an increase in dielectric permittivity in PANI- $\mathrm{Fe}_{3} \mathrm{O}_{4}$, synthesized by a self-assembly process of $\mathrm{Fe}_{3} \mathrm{O}_{4}$ nanoparticles with polyaniline. ${ }^{37}$ It must be noted here that unlike poly pyrrole, PANI is composed of both quinoid and benzenoid rings units. It is reported that PANI- $\mathrm{Fe}_{3} \mathrm{O}_{4}$ core-shell structure possesses a higher ratio of benzenoid ring when compared to pure PANI. ${ }^{37}$ The bonding between ferrite particles and polyaniline arises out by both the coordination of ferric ion with the nitrogen atom of quinine rings in PANI and hydrogen bonding between the oxygen of $\mathrm{Fe}_{3} \mathrm{O}_{4}$ and the -NH of PANI. ${ }^{35,37}$ However, we adopted a method of radio frequency (rf) plasma polymerization to passivate $\mathrm{Fe}_{3} \mathrm{O}_{4}$ nano particles. The employment of $\mathrm{rf}$ polymerization to make core-shell particles differs with respect to in situ chemical routes. We have earlier reported that extended conjugation occurs during rf polymerization. ${ }^{38}$
We recently reported enhancement of magnetization in a core-shell architecture consisting of $\mathrm{Fe}_{3} \mathrm{O}_{4} / \gamma-\mathrm{Fe}_{2} \mathrm{O}_{3}$ core and a polyaniline shell. ${ }^{39}$ The enhanced magnetization was explained based on a contact potential induced phenomenon. Later, we focused our studies on the dielectric properties of this core-shell architecture, and these results are being reported in this communication. Commercial samples of $\mathrm{Fe}_{3} \mathrm{O}_{4}$ lying in the range of $25-30 \mathrm{~nm}$ are subjected to $\mathrm{RF}$ plasma polymerization to create an appropriate organic thin layer over it. Structural characterization of the samples was carried out using X-ray diffraction. The core shell structure of iron oxide-PANI was confirmed by using Transmission Electron Microscope (TEM) and High Resolution Transmission Electron Microscope (HRTEM). Dielectric permittivity of core-shell structure has been investigated by dielectric spectroscopic methods using an HP 4285A LCR meter. The role of an appropriate model in explaining the observed permittivity enhancement was also studied. The details and schematic diagram of the experimental set up for the surface passivation of magnetic iron oxide by radio frequency polymerization are described elsewhere. ${ }^{39}$

Uncoated and PANI coated powdered samples were structurally characterized by using a Rigaku D-Max-C X ray diffractometer with an incident $\mathrm{Cu} \mathrm{K}_{\alpha}$ radiation of $1.54 \mathrm{~A}^{\mathrm{o}}$ at $30 \mathrm{kV}$ and $20 \mathrm{~mA}$. Transmission Electron Microscopy was used to estimate particle size distribution of nanoparticles. The dielectric permittivity measurements of both PANI coated and uncoated iron oxide nano particles at different frequencies from $100 \mathrm{KHz}$ to $8 \mathrm{MHz}$ were carried out by a Hewlett Packard 4285A LCR meter, where the data acquisition and its analysis are completely automated and controlled by a virtual instrumentation package called LabVIEW supplied by National Instruments, wherein more than 20000 data points can be acquired in a matter of few minute.

Figure 1 shows the XRD patterns of both uncoated and PANI coated magnetite. Both the XRD patterns match with ICDD [ICDD No.85-1436] values for the spinel magnetite which corresponds to the Fd3m space group. No extra peaks corresponding to any impurities were observed. It has been found that there is an overall decrease in intensities of the corresponding peaks of PANI coated samples. This indicates effective surface modification of nanoparticles with PANI.

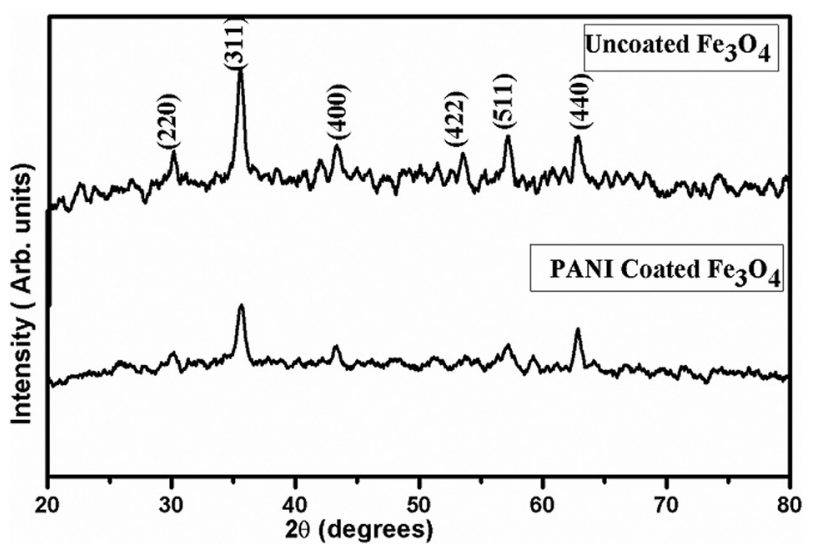

FIG. 1. XRD pattern of PANI coated and uncoated magnetite nanoparticles. Reprinted with permission from Sethulakshmi et al., Applied Physics Letters. 103, 162414 (2013). Copyright 2013 American Institute of Physics. ${ }^{39}$ 

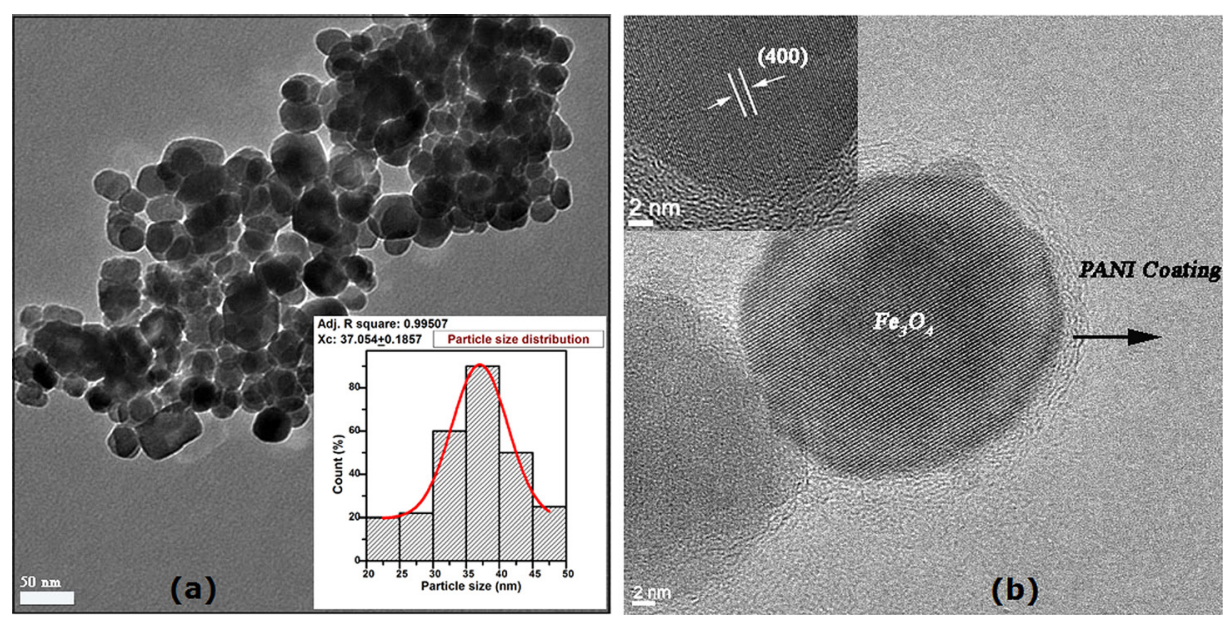

FIG. 2. (a) TEM image of PANI coated $\mathrm{Fe}_{3} \mathrm{O}_{4}$ nanoparticles. Inset shows particle size distribution. (b) HRTEM images of PANI coated single $\mathrm{Fe}_{3} \mathrm{O}_{4}$ particle with core shell structure. Inset shows HRTEM image correspond to the lattice plane (400). Reprinted with permission from Sethulakshmi et al., Applied Physics Letters 103, 162414 (2013). Copyright 2013 American Institute of Physics. ${ }^{39}$

The average crystallite size of uncoated $\mathrm{Fe}_{3} \mathrm{O}_{4}$ estimated by using peak broadening technique with Scherrer's formula is found to be $30 \mathrm{~nm}$. Figure 2(a) shows a TEM micrograph of the polymer coated $\mathrm{Fe}_{3} \mathrm{O}_{4}$ nano powder from which average particle size can be determined. It can be seen from HRTEM images in Figure 2(b) that a magnetite nano particle of diameter $30 \mathrm{~nm}$ as core is surrounded by a $4 \mathrm{~nm}$ thickness of polyaniline as shell forming a core shell structure. The average particle size obtained from TEM was estimated to be $36-40 \mathrm{~nm}$. The inter planar spacing (d) estimated from the HRTEM image correspond to the lattice plane (400) which is indexed in the inset of Figure 2(b).

The frequency response of $\mathrm{Fe}_{3} \mathrm{O}_{4}$ before PANI coating carried out using LCR meter is shown in Figure 3. It can be noticed that the value of dielectric permittivity of the samples decreases with increase in frequency. At higher frequencies, the dipoles are unable to respond swiftly to follow the applied field, and, consequently, the dipole polarization decreases and leads to smaller dielectric values. This trend of decrease of dielectric permittivity with frequency is observed for PANI coated magnetite samples also.

It is interesting to note that there is a significant enhancement of dielectric permittivity for the PANI- $\mathrm{Fe}_{3} \mathrm{O}_{4}$ core shell samples with respect to uncoated ones. This enhancement of dielectric permittivity can be correlated to the onset of Maxwell-Wagner behavior due to interfacial polarization. Because of interfacial effects, charge carriers are trapped at

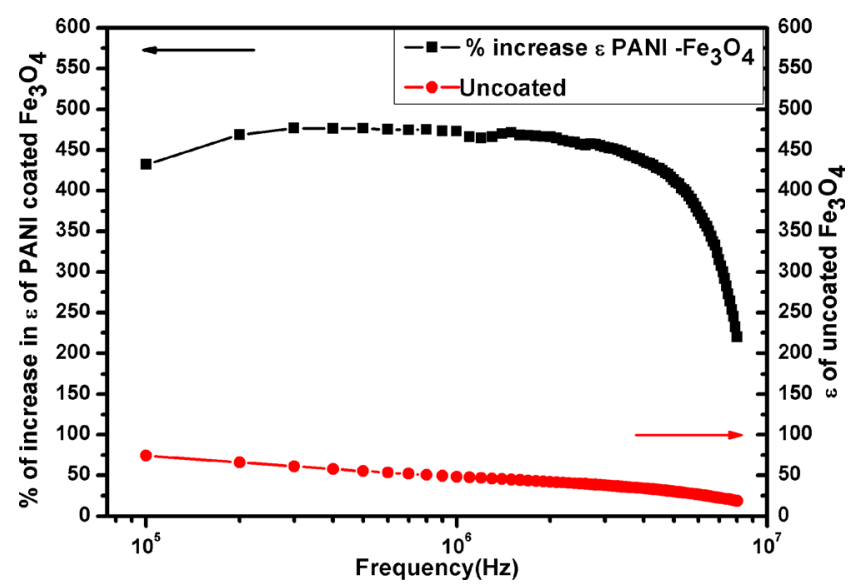

FIG. 3. Real dielectric permittivity of uncoated $\mathrm{Fe}_{3} \mathrm{O}_{4}$ and percent $(\%)$ of increase in $\varepsilon$ of $\mathrm{PANI}-\mathrm{Fe}_{3} \mathrm{O}_{4}$. inclusions of PANI in the dielectric medium of $\mathrm{Fe}_{3} \mathrm{O}_{4}$ giving rise to conducting regions. This results in an effective polarization, and also the behavior of dielectric permittivity with respect to frequency is similar to that of orientational polarization. Percentage of increase in real dielectric permittivity $(\varepsilon)$ of $\mathrm{PANI}-\mathrm{Fe}_{3} \mathrm{O}_{4}$ with respect to uncoated one is shown in Figure 3. The loss tangent $(\tan \delta)$ indicating the dielectric loss of both uncoated and PANI- $\mathrm{Fe}_{3} \mathrm{O}_{4}$ as a function of frequency is depicted in the inset of Figure 4. It can be seen that the dielectric loss of coated $\mathrm{Fe}_{3} \mathrm{O}_{4}$ exhibits a higher value in the range less than $500 \mathrm{kHz}$. However, the dielectric loss of PANI coated magnetite decreases and remains at lower values with respect to uncoated sample for a wide range of frequency greater than $500 \mathrm{kHz}$. Higher dielectric loss of PANI coated $\mathrm{Fe}_{3} \mathrm{O}_{4}$ in the lower frequency regime $(<500 \mathrm{kHz})$ could be emanating from charge accumulation in the dielectric interface due to space charge polarization. ${ }^{40}$

Maxwell-Wagner capacitor model can be applied to this heterogeneous medium so that the effective polarization is determined by the accumulation of charges at discontinuous interfaces. In order to explain the enhanced dielectric permittivity observed after PANI coating, the experimental results are analyzed in terms of a simplified model using Maxwell-Wagner effect. $^{20,21}$

The real and imaginary parts of relative permittivity by Maxwell-Wagner capacitor are given by

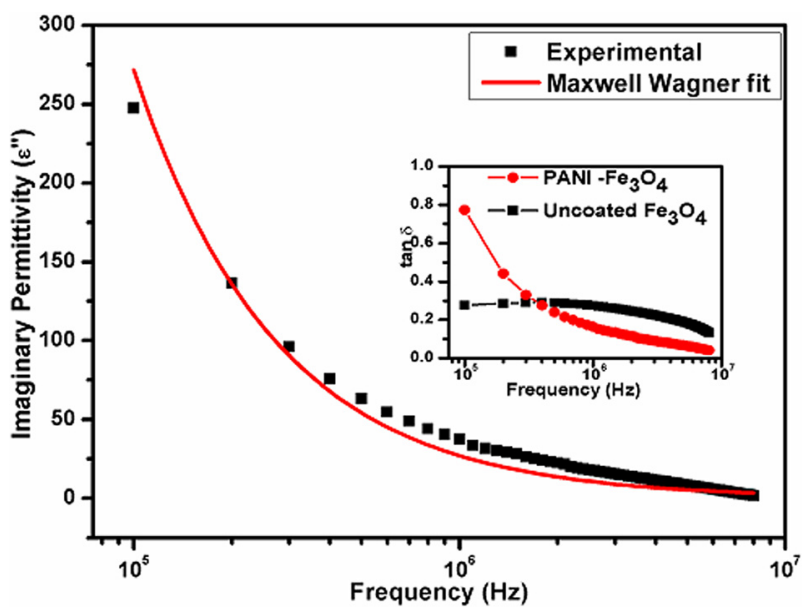

FIG. 4. Maxwell-Wagner fit to imaginary permittivity of $\mathrm{PANI}-\mathrm{Fe}_{3} \mathrm{O}_{4}$. Inset shows dielectric loss tangent of uncoated $\mathrm{Fe}_{3} \mathrm{O}_{4}$ and $\mathrm{PANI}-\mathrm{Fe}_{3} \mathrm{O}_{4}$. 


$$
\begin{gathered}
\varepsilon^{\prime}(\omega)=\frac{1}{C_{0}\left(R_{1}+R_{2}\right)} \frac{\tau_{1}+\tau_{2}-\tau+\omega^{2} \tau_{1} \tau_{2} \tau}{1+\omega^{2} \tau}, \\
\varepsilon^{\prime \prime}(\omega)=\frac{1}{\omega C_{0}\left(R_{1}+R_{2}\right)} \frac{1-\omega^{2} \tau_{1} \tau_{2}+\omega^{2} \tau\left(\tau_{1}+\tau_{2}\right)}{1+\omega^{2} \tau},
\end{gathered}
$$

where $\tau_{1}, \tau_{2}$, and $\tau$ are, respectively, the dielectric relaxation times of coating layer, core material, and that of core-shell. $\mathrm{C}_{0}$ is a geometric factor and $\mathrm{R}_{1}$ and $\mathrm{R}_{2}$ are resistances of the coating layer and core.

The limiting cases for Eq. (1) at zero and infinite frequency are same as that for Debye relaxation. So, for diagnosing interfacial polarization effects, analysis of imaginary part of relative permittivity is preferred rather than the real part. On applying limiting conditions, Eq. (2) takes the form

$$
\varepsilon^{\prime \prime}(\omega)=\frac{1}{\omega C_{0}\left(R_{1}+R_{2}\right)}+\frac{\left(\varepsilon_{0}-\varepsilon_{\infty}\right) \omega \tau}{1+\omega^{2} \tau} .
$$

Thus, to distinguish Maxwell-Wagner behavior from Debye mechanism, imaginary permittivity vs frequency curve is fitted as per Maxwell-Wagner model given by Eq. (3). From Figure 4, it can be demonstrated that frequency response of imaginary permittivity can be directly modeled by Maxwell-Wagner expression.

The $\mathrm{Fe}_{3} \mathrm{O}_{4}$-PANI core shell structure forms an inhomogeneous medium with the core and the outer shell having different dielectric permittivity. The effective field is different for the coated particles, and this field causes an interfacial polarization between the grain boundaries and the polymer layer in contact with the particle surface according to the Koop's phenomenon. ${ }^{41}$ This polarization causes an increase in the dielectric susceptibility of the system and leads to dielectric enhancement.

Since the core shell structure contains regions of different permittivity, the specimen is inhomogeneous which is similar to a composite arrangement consisting of two parallel-sided slabs of materials with different properties. It is possible to calculate the apparent dielectric permittivity of the core shell structure in terms of coating thickness by a simplified model using effective medium theory of Maxwell-Wagner and Bruggeman. ${ }^{29-31}$ For that, PANI- $\mathrm{Fe}_{3} \mathrm{O}_{4}$ can be assumed as a spherical nano capacitor consisting of two layers of different dielectric constant and thickness values. The apparent dielectric permittivity ${ }^{42}\left(\varepsilon_{T}\right)$ can be calculated as

$$
\varepsilon_{T}=\frac{\varepsilon_{2}\left(\varepsilon_{1}-j 4 \pi \sigma / \omega \varepsilon\right)}{\left(\varepsilon_{1}-j 4 \pi \sigma / \omega \varepsilon\right) f_{2}+\varepsilon_{2} f_{1}} .
$$

Here, $\varepsilon_{T}$, is the apparent dielectric constant, $\sigma$ is the conductivity of the medium of dielectric constant $\varepsilon_{1}$ (coating layer), and $\varepsilon_{2}$ is the dielectric constant of the core material. Here, $f_{1}=d_{1} /\left(d_{1}+d_{2}\right)$ and $f_{2}=d_{2} /\left(d_{1}+d_{2}\right)$, where $d_{1}$ is the thickness of the coating and $d_{2}$ is the diameter of the core.

Thus in addition to the permittivity values of the core and the coating, the particle size of core and the thickness of the coating also influence the value of dielectric permittivity. The apparent permittivity for a $4 \mathrm{~nm}$ coating thickness on core using real part of Eq. (4) was calculated. Figure 5 shows calculated as well as measured values of the apparent

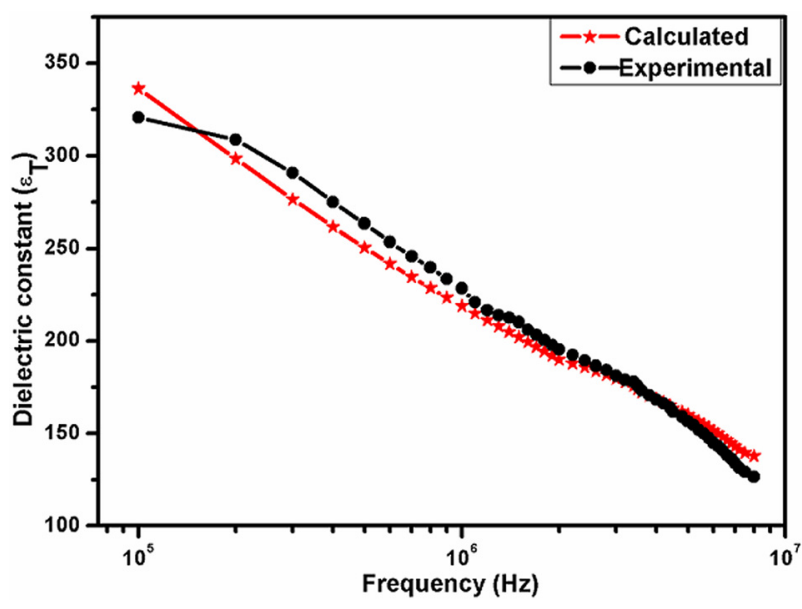

FIG. 5. Calculated and experimental value of apparent dielectric constant vs frequency of PANI- $\mathrm{Fe}_{3} \mathrm{O}_{4}$ with $4 \mathrm{~nm}$ coating thickness.

dielectric constant of PANI- $\mathrm{Fe}_{3} \mathrm{O}_{4}$ with $4 \mathrm{~nm}$ coating thickness. It can be observed from the plot that, the experimentally observed values agree well with the calculated values of $\varepsilon_{T}$.

The dielectric permittivity of polymer coated $\mathrm{Fe}_{3} \mathrm{O}_{4}$ has significantly enhanced from 74 to 320 ( $\sim 4$ times as that of uncoated one). Most reports regarding this kind of large enhancement of dielectric permittivity is explained by means of Maxwell-Wagner extrinsic effects rather than the intrinsic features of the bulk. Earlier, Lunkenheimer et al. reported the formation of a high capacitive depletion layer due to Schottky barriers at the interfaces of metal-to insulator contacts/inter grain boundaries leading to the suppression of electron concentration in the contact region of semiconductor. ${ }^{27}$ Later, Wu et al. have interpreted the large dielectric permittivity (approximately two orders of magnitude) observed for $\mathrm{BiFeO}_{3}$ when doped with $\mathrm{Mg}$ by Maxwell-Wagner relaxation based on IBLC, wherein the Maxwell-Wagner polarization effects built up in the insulating grain boundaries between the semiconducting grains of polycrystalline $\mathrm{BiFeO}_{3}$ which generates high dielectric permittivity. ${ }^{24}$ But, the combined contribution of Schottky and IBLC Maxwell-Wagner effects was suggested by Krohns for the successful explanation of the colossal dielectric constants observed in surface modified $\mathrm{CaCuTi}_{4} \mathrm{O}_{12}$ ceramic with gold sputtering. ${ }^{26}$ They assumed that gold sputtered over semiconductor forms a metal covering a thin insulating layer on top of a semiconductor, which leads to metal-insulator-semiconductor (MIS) diodes. Our core shell structure of $\mathrm{Fe}_{3} \mathrm{O}_{4}$-PANI is electrically heterogeneous like above mentioned cases. Though different models were invoked to explain enhancement of dielectric permittivity on different systems, from a mere dielectric permittivity study, it is hard to pin point a specific mechanism in our case, and any attempt to do so will be speculative without further systematic studies.

RF plasma polymerization technique was employed for surface passivation of $\mathrm{Fe}_{3} \mathrm{O}_{4}$ nano particles to arrive at a core shell structure. The influence of conducting polymer coating on the electrical behaviour of core nano particles were investigated by dielectric spectroscopic methods. A significant enhancement in the dielectric permittivity was 
observed for surface modified iron oxides when compared with uncoated ones. This enhancement of dielectric permittivity by surface modification was modeled using Maxwell-Wagner theory which predicts both the dielectric enhancement and frequency dependence. Both the theory and experimental values seem to be in good agreement. The influence of coating thickness on the effective dielectric permittivity of electrically heterogeneous core-shell was also analyzed by using a simplified model of effective medium theory of Maxwell-Wagner and Bruggeman. The possibility of barrier mechanisms such as IBLC and SBLC in the large enhancement of dielectric permittivity of $\mathrm{Fe}_{3} \mathrm{O}_{4}$ after PANI coating was discussed. The observed large dielectric permittivity exhibited by $\mathrm{Fe}_{3} \mathrm{O}_{4}$-PANI core shell architecture is quite interesting not only from a fundamental perspective but also from an application point of view. The influence of layer thickness on the dielectric permittivity is also of interest.

L.K.J. acknowledges UGC RFSMS (No. F.4-1/2006 (BSR)/5-22/2007) for providing financial assistance and M.R.A. acknowledges DAE BRNS for funding provided in the form of Project (No. 2011/34/7/BRNS/0596).

${ }^{1}$ S. Kalele, S. W. Gosavi, J. Urban, and S. K. Kulkarni, Curr. Sci. 91, 1038-1052 (2006), available at http://www.currentscience.ac.in/Downloads/ download_pdf.php?titleid=id_091_08_1038_1052_0.

${ }^{2}$ A. H. Faraji and P. Wipf, Bioorg. Chem. 17, 2950-2962 (2009).

${ }^{3}$ V. Sunny, T. N. Narayanan, U. S. Sajeev, P. A. Joy, D. S. Kumar, Y. Yoshida, and M. R. Anantharaman, Nanotechnology 17, 4765 (2006).

${ }^{4}$ A. K. Gupta and M. Gupta, Biomaterials 26, 3995-4021 (2005).

${ }^{5}$ A. P. R. Mary, T. N. Narayanan, V. Sunny, D. Sakthikumar, Y. Yoshida, P. A. Joy, and M. R. Anantharaman, Nanoscale Res. Lett. 5, 1706-1711 (2010).

${ }^{6}$ H.-T. Jeng and G. Y. Guo, Phys. Rev. B 65, 094429-094438 (2002).

${ }^{7}$ H. Zeng, S. Sun, J. Li, Z. L. Wang, and J. P. Liu, Appl. Phys. Lett. 85, $792-794$ (2004).

${ }^{8}$ S. Yamamuro, K. Yamamoto, D. L. Peng, T. Hirayama, and K. Sumiyama, Appl. Phys. Lett. 90, 242510 (2007).

${ }^{9}$ J. K. Yuan, S. H. Yao, Z. M. Dang, A. Sylvestre, M. Genestoux, and J. B. Bai, J. Phys. Chem. C. 115, 5515-5521-(2011).

${ }^{10}$ P. Barber, S. Balasubramanian, Y. Anguchamy, S. Gong, A. Wibowo, H. Gao, H. J. Ploehn, and H.-C. zur Loye, Materials 2, 1697-1733 (2009).

${ }^{11}$ M. Guo, T. Hayakawa, M. Kakimoto, and T. Goodson, J. Phys. Chem. B 115, 13419-13432-(2011).

${ }^{12}$ Z. M. Dang, S. H. Yao, J. K. Yuan, and J. Bai, J. Phys. Chem. C 114, 13204-13209- (2010).

${ }^{13}$ L. Xie, X. Huang, Y. Huang, K. Yang, and P. Jiang, J. Phys. Chem. C 117, 22525-22537-(2013).
${ }^{14}$ G. Bidan, O. Jarjayes, J. M. Fruchart, and E. Hanneeart, Adv. Mater. 6, 152 (1994).

${ }^{15}$ L. Gai, X. Han, Y. Hou, J. Chen, H. Jiang, and X. Chen, Dalton Trans. 42, 1820 (2013).

${ }^{16}$ O. Jarjayes, P. H. Fries, and G. Bidan, Synth. Met. 69, 343 (1995).

${ }^{17}$ Y. Xiaotun, X. Lingge, N. S. Choon, and C. S. O. Hardy, Nanotechnology 14, 624-629 (2003).

${ }^{18}$ H. Tabata, H. Tanaka, and T. Kawai, Appl. Phys. Lett. 65, 1970-1972 (1994).

${ }^{19}$ D. H. Liu, C. Xu, and P. M. Hui, Appl. Phys. Lett. 92, 181901-181903 (2008).

${ }^{20}$ M. Shen, S. Ge, and W. Cao, J. Phys. D: Appl. Phys. 34, 2935-2938 (2001).

${ }^{21}$ D. O’Neill, R. M. Bowman, and J. M. Gregg, Appl. Phys. Lett. 77, 1520-1522 (2000).

${ }^{22}$ G. Catalan, D. O’Neill, R. M. Bowman, and J. M. Gregg, Appl. Phys. Lett. 77, 3078-3080 (2000).

${ }^{23}$ Z. Yu and C. Ang, J. Appl. Phys. 91, 794-797 (2002).

${ }^{24}$ H. Wu, Y. B. Lin, J. J. Gong, F. Zhang, M. Zeng, M. H. Qin, Z. Zhang, Q. Ru, Z. W. Liu, X. S. Gao, and J. M. Liu, J. Phys. D: Appl. Phys. 46, 145001-145005 (2013).

${ }^{25}$ D. C. Sinclair, T. B. Adams, F. D. Morrison, and A. R. West, Appl. Phys. Lett. 80, 2153-2155 (2002).

${ }^{26}$ S. Krohns, P. Lunkenheimer, S. G. Ebbinghaus, and A. Loidl, J. Appl. Phys. 103, 084107-084115 (2008).

${ }^{27}$ P. Lunkenheimer, V. Bobnar, A. V. Pronin, A. I. Ritus, A. A. Volkov, and A. Loidl, Phys. Rev. B 66, 052105-052108 (2002).

${ }^{28}$ P. Fiorenza, R. L. Nigro, V. Raineri, G. Malandrino, R. G. Toro, and M. R. Catalano, J. Appl. Phys. 108, 074103-074107 (2010).

${ }^{29}$ D. A. C. Bruggeman, Ann. Phys. (Leipzig) 416, 636 (1935).

${ }^{30}$ C. G. Granqvist and O. Hunderi, Phys. Rev. B 18, 1554-1561 (1978).

${ }^{31}$ Y. Rao, J. Qu, T. Marinis, and C. P. Wong, IEEE Trans. Compon. Packag. Technol. 23, 680-683 (2000).

${ }^{32}$ S. Pal and E. C. Alocilja, Biosens. Bioelectron. 24, 1437-1444 (2009).

${ }^{33}$ S. Saravanan, C. J. Mathai, M. R. Anantharaman, and S. Venkatachalam, New J. Phys. 6, 64 (2004).

${ }^{34}$ M. Wan and W. Li, J. Polym. Sci. Polym. Chem. 35, 2129 (1997).

${ }^{35}$ G. Qiu, Q. Wang, and M. Nie, J. Appl. Polym. Sci. 102, 2107-2111 (2006).

${ }^{36}$ B. Z. Tang, Y. Geng, Q. Sun, X. X. Zhang, and X. Jing, Pure Appl. Chem. 72, 157-162 (2000).

${ }^{37}$ S. K. El-Mahy, M. Dawy, and E. Abd El Aziz, J. Appl. Sci. Res. 9(4), 2918-2926 (2013), available at http://www.aensiweb.com/jasr/jasr_april_ 2013.html, then click on the page range 2918-2926.

${ }^{38}$ U. S. Sajeev, C. J. Mathai, S. Saravanan, R. R. Ashokan, S. Venkatachalam, and M. R. Anantharaman, Bull. Mater. Sci. 29, 159-163 (2006).

${ }^{39}$ N. Sethulakshmi, V. Sooraj, U. S. Sajeev, S. S. Nair, T. N. Narayanan, L. K. Joy, P. A. Joy, P. M. Ajayan, and M. R. Anantharaman, Appl. Phys. Lett. 103, 162414-162418 (2013).

${ }^{40}$ R. Ravindran, K. Gangopadhyay, S. Gangopadhyay, N. Mehta, and N. Biswas, Appl. Phys. Lett. 89, 263511 (2006).

${ }^{41}$ C. G. Koops, Phys. Rev. 83, 121-124 (1951).

${ }^{42}$ N. E. Hill, Dielectric Properties and Molecular Behaviour (Van Nostrand Reinhold, New York, 1969). 